\title{
The molecular basis for the differential regulation of the hlyE-encoded haemolysin of Escherichia coli by FNR and HlyX lies in the improved Activating Region 1 contact of HlyX
}

\author{
Jeffrey Green and Mandy L. Baldwin
}

Department of Molecular Biology \& Biotechnology, University of Sheffield, Western Bank, Sheffield S10 2TN, UK Author for correspondence: Jeffrey Green. Tel: +44114222 4403. Fax: +44 1142728697.
e-mail: jeff.green@sheffield.ac.uk

The regulator of fumarate and nitrate reduction (FNR) protein of Escherichia coli is an oxygen-responsive transcription regulator that acts mainly to activate the transcription of genes associated with anaerobic energy generation during periods of oxygen starvation. The hlyX gene of the swine pathogen Actinobacillus pleuropneumoniae encodes an FNR homologue, HlyX, which can complement the anaerobic respiratory deficiencies of an fnr mutant. However, FNR and HlyX have distinct but overlapping regulons because during anaerobic incubation, $h / y X$-expressing $E$. coli K-12 strains produce an otherwise latent haemolysin. The gene encoding the 'latent' haemolysin has been designated $h / y E$ and analysis of the promoter region by DNase I footprinting reveals the presence of an FNR- (HlyX-) binding site. Anaerobic expression of an hlyE:: lacZ reporter was 6.5-fold higher in hlyX compared to fnr-expressing cells. Both FNR and HlyX recruited RNA polymerase to the hlyE promoter but formed different ternary complexes. One major transcript (tsp1) initiating at 78.5 bp downstream of the FNR-binding site and four minor transcripts initiating at 73.5 (tsp2), 71.5 (tsp3), 63.5 (tsp4) and 62.5 (tsp5) bp from the FNR site were detected. From the position of the FNR box relative to the transcript starts, hlyE is expressed from a Class I FNR-regulated promoter. Substitution of selected FNR amino acids with the residues found in the equivalent positions in HlyX indicated that Activating Region 1 (AR1) of FNR forms a surface encompassing $\beta_{9}$ to $\beta_{11}$ and that the AR1 contact at Class I promoters is different to that at Class II promoters, although the same surface is involved. The FNR variant, FNR-A225T, combined the properties of FNR (good activation from Class II promoters) and HlyX (good activation of Class I promoters) and conferred the haemolytic phenotype.

Keywords: FNR, HlyX, HlyE, Class I promoter, haemolysin

\section{INTRODUCTION}

The regulator of fumarate and nitrate reduction (FNR) protein of Escherichia coli is an oxygen-responsive member of the cAMP receptor protein (CRP) family of transcription regulators. All the members of this family are structurally related to CRP and, with the exception of the Bacillus subtilis FNR (Ramos et al., 1995), have a C-terminal helix-turn-helix DNA-binding domain and an N-terminal sensory domain (Guest et al., 1996). The

Abbreviations: AR1, Activating Region 1; CRP, CAMP receptor protein; FNR, regulator of fumarate and nitrate reduction.
blyX gene of the pig pathogen Actinobacillus pleuropneumoniae encodes an FNR protein, known as HlyX, that is $73 \%$ identical to E. coli FNR at the amino acid level. Both FNR and HlyX sense oxygen starvation through the anaerobic acquisition of an oxygen-labile [4Fe-4S] cluster (Lazazzera et al., 1996; Green et al., 1996a, b; Green \& Baldwin, 1997). Incorporation of the iron-sulphur cluster promotes dimerization of FNR and enhances binding to the FNR (HlyX) DNA target, TTGAT---ATCAA (Lazazzera et al., 1996; Green \& Baldwin, 1997).

Unsurprisingly, HlyX is able to complement the anaerobic growth deficiencies of an $f n r$ mutant. However, 
HlyX also anaerobically activates the expression of a number of genes in E. coli $\mathrm{K}-12$ strains, including a haemolysin, that FNR apparently does not (MacInnes et al., 1990; Green et al., 1992; Green \& Baldwin, 1997). Thus FNR and HlyX have distinct but overlapping modulons. The construction of FNR-HlyX hybrid proteins led to the proposal that the endowment of the haemolytic phenotype required unique features of both the $\mathrm{N}$ - and C-terminal regions of HlyX (Green et al., 1992). Recently, the $N$-terminal component has been shown to reside in the $\beta_{9}-\beta_{10}$ loop (Activating Region 1 , AR1) of HlyX and is important in transcription activation from Class I promoters. Thus it was predicted that the promoter region of the 'latent haemolysin' gene would contain an FNR box located at -61 or beyond (Green \& Baldwin, 1997).

Recently, an E. coli gene, designated hlyE, has been identified. It is located at $26.4 \mathrm{~min}$ and encodes a protein ( $34 \mathrm{kDa})$ which, when overproduced, confers a haemolytic phenotype upon E. coli (GenBank accession no. U57430). The molecular mass of the product is the same as the previously described cryptic contact-dependent haemolysin ClyA (cytolysin A), which is secreted by some strains of $E$. coli $\mathrm{K}-12$, including $h n s$ mutants and strains overexpressing $c l y A$ and $\operatorname{sly} A$ (Oscarsson $e$ t al., 1996). Although the $c l y A$ sequence is unavailable, it is likely that $\operatorname{cly} A$ is identical to $h l y E$. The slyA gene is thought to encode a regulator of cytolysin $\mathrm{A}$ expression (Oscarsson et al., 1996). SlyA is homologous to several known regulatory proteins but the nature of the SlyA stimulus and response is unknown.

In this study hlyE expression is shown to be driven from a Class I FNR-dependent promoter that is differentially regulated by FNR and HlyX. Single substitutions of amino acids in the $\beta_{9}-\beta_{10}$ region of FNR with those present in HlyX generally resulted in improved activation from the $h l y E$ promoter and two model Class I promoters, but this was not sufficient in itself to confer a haemolytic phenotype. A substitution in $\beta_{11}$ resulted in an FNR which combined the properties of FNR (good Class II activation) and HlyX (good Class I activation) and allowed the detection of the haemolytic phenotype.

\section{METHODS}

Plasmids and bacterial strains. Plasmids used to test the effects of the anaerobic expression of $f n r, b l y X$ and the $f n r$ and $b l y X$ mutants were the ptac85 derivatives: pGS330, FNR (Green et al., 1991); pGS415, HlyX (Green et al., 1992); pGS1003, HlyX-A187P (Green \& Baldwin, 1997); pGS1053, FNRR179Q; pGS1054, FNR-F181Y; pGS1055, FNR-Q183A; pGS1056, FNR-R184P; pGS1057, FNR-P188A ; pGS1086, FNR-A225T; pGS1085, FNR-Cdel17, which lacks 17 Cterminal amino acids; pGS1094, FNR-Cdel10, which lacks 10 C-terminal amino acids. Western blotting with anti-FNR serum indicated that expression of all the FNR derivatives was similar to that observed with FNR. However, the products of pGS1085, FNR-Cdel17 and pGS1094, FNR-Cdel10 were insoluble.

The source of the $h l y E$ promoter DNA used in the footprinting analyses was pGS1051 constructed by ligating a PCR-gener- ated blyE promoter fragment $(-97$ to +61$)$ into EcoRI-BamHI-digested pUC118.

The ability of FNR, HlyX and altered FNR and HlyX proteins to activate transcription from hlyE and model Class $I$ and Class II promoters was determined using: pGS1065, a pRW50 derivative, constructed by ligating the EcoRI-BamHI blyE promoter-containing fragment from pGS1051 into pRW50; and the semi-synthetic lacZ reporter fusions, FFpmelR (Class II, FNR site at $-41 \cdot 5$ ), FF+20pmelR (Class I, FNR site at -61.5 ) and $F F-71.5 p m e l R$ (Class I, FNR site at -71.5 plus an improved -35 element) (Wing et al., 1995) in JRG1728 $\left[\Delta(\right.$ laclPOZYA $) \quad \chi^{74}$ galU galk rpsL $\Delta($ ara-leu $)$ $\Delta($ tyrR-fnr-rac-trg) $17 z d d-230:$ : Tn9] containing pGS330 to generate JRG3653, 3402, 3403 and 3404, pGS415 to generate JRG3654, 3405, 3206 and 3407, pGS1003 to generate JRG3703, 3452,3453 and 3454 , pGS1053 to generate JRG3704, 3657, 3655 and 3656 , pGS1054 to generate JRG3705, 3660, 3658 and 3659 , pGS1055 to generate JRG3706, 3663, 3661 and 3662, pGS1056 to generate JRG3739, 3735, 3737 and 3736, pGS1057 to generate JRG3707, 3666, 3664 and 3665 , pGS1086 to generate JRG3749, 3745,3747 and 3746 or pGS1094 to generate JRG3761, 3762, 3763, and 3764. JRG3702 is JRG1728(pGS1065). The authenticity of pGS1065 was confirmed by automated DNA sequencing. Cultures were grown aerobically in $\mathrm{L}$ broth with vigorous shaking or anaerobically in $\mathrm{L}$ broth supplemented with $0.4 \%$ glucose to an $\mathrm{OD}_{600}$ of $0.3-0.5$ (Pye Unicam UV4) in sealed bottles at $37^{\circ} \mathrm{C} . \beta$ Galactosidase activity was determined according to Miller (1972).

DNase I footprinting. DNase I footprinting was carried out as described previously (Green et al., 1991) except that the reactions were performed anaerobically and contained (in a total volume of $10 \mu \mathrm{l}$ ) : hlyE promoter DNA (approximately $10 \mathrm{ng}$ ), end-labelled at the BamHI site (top strand) of the PvuI-BamHI fragment of pGS1051 (275 bp); HlyX or FNR $(0-100 \mathrm{nM})$ or RNA polymerase $(0 \cdot 2-1 \mathrm{U}) ; 2 \mu \mathrm{l} 5 \times$ binding buffer $(5 \times$ binding buffer is $0.1 \mathrm{M}$ Tris $/ \mathrm{HCl}, \mathrm{pH} 8.0 ; 0.05 \mathrm{M}$ $\mathrm{MgCl}_{2} ; 50 \mathrm{mM}$ dithiothreitol; $25 \%$, v/v, glycerol). The mixtures were incubated for $2 \mathrm{~min}$ at $25^{\circ} \mathrm{C}$ followed by digestion with DNase I ( $1 \mu \mathrm{l}$ of $1 \mathrm{U} \mathrm{ml}^{-1}$ for $15-60 \mathrm{~s}$ at $\left.25^{\circ} \mathrm{C}\right)$. Reactions were stopped by addition of $200 \mu \mathrm{l} 0.3 \mathrm{M}$ sodium acetate containing $10 \mathrm{mM}$ EDTA followed by phenol/ chloroform extraction. The DNA was ethanol-precipitated and resuspended in $10 \mu \mathrm{l}$ loading buffer $(40 \%, \mathrm{v} / \mathrm{v}$, formamide; $5 \mathrm{M}$ urea ; $5 \mathrm{mM} \mathrm{NaOH}$; $1 \mathrm{mM}$ EDTA ; 0.03\% bromophenol blue; $0.03 \%$ xylene cyanol) for electrophoretic fractionation on polyacrylamide-urea gels and autoradiographic analysis. A Maxam \& Gilbert G track of the same fragment was used to provide a calibration (Maxam \& Gilbert, 1980).

Transcript mapping. The transcription start point of the blyE promoter was determined by primer extension. Total RNA was prepared from JRG2269 ( f $n r$ hly $\left.X^{+}\right)$, JRG3350 (fnr $r^{+}$after anaerobic growth in the presence of $30 \mathrm{mg}$ IPTG $1^{-1}$, or from MC4100, JRG1728 (fnr), RH90 (rpoS), SJP3 (ihfA), RJ1802 (fis) or ECL585 ( $\operatorname{arcA}$ ) after anaerobic growth in the absence of IPTG, as described by Aiba et al. (1981). For primer extension, the method of Gerischer \& Durre (1992) was used with 10 pmol primer S420 (CAGTCATAATCATTCGCCTC, blyE co-ordinates 154-135), approximately $100 \mu \mathrm{g}$ RNA and $50 \mathrm{U}$ AMV reverse transcriptase $(\mathrm{Nbl})$. After ethanol precipitation, the DNA was fractionated on calibrated ureapolyacrylamide gels and analysed by autoradiography.

Other methods. FNR and HlyX were purified from the respective GST-fusion proteins and their [4Fe-4S] clusters reconstituted as described previously (Green et al., 1996a, b; Green \& Baldwin, 1997). The altered FNR proteins used in 
this study were generated using the Altered Sites II System (Promega) with the appropriate mutagenic oligonucleotides and presence of the desired substitutions was confirmed by automated DNA sequencing.

In vitro transcription was as described previously (Green et al., 1996c) except $n d h$ DNA was replaced by $h l y E$ (PvuI-BamHI fragment from pGS1051) and Nbp was replaced by FNR or HlyX. Gels were calibrated with a Maxam \& Gilbert $G$ track.

The ability of FNR, HlyX and the altered HlyX proteins to confer a haemolytic phenotype was assessed after anaerobic

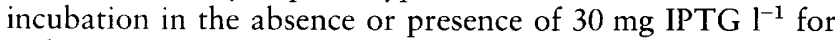
$24 \mathrm{~h}$ at $37^{\circ} \mathrm{C}$ on blood agar $(7 \%, \mathrm{w} / \mathrm{v}$, horse blood) of E. coli JRG1728 expressing $f n r, h l y X$ or the mutant $f n r$ or $h l y X$ genes from the tac promoter of ptac85 (Green et al., 1992).

Western blotting with anti-FNR serum was as described by Spiro \& Guest (1987).

\section{RESULTS}

\section{The hlyE promoter contains an FNR site and is differentially regulated by FNR and HlyX}

Analysis of the DNA upstream of the $h l y E$ coding region revealed the presence of an FNR-binding site which matches the consensus in 9 out of 10 bases (Fig. 1). The FNR site was shown to be functional by estimating the anaerobic induction of $\beta$-galactosidase activity from a blyE: :lacZ reporter plasmid (pGS1065) in the $f n r$ strain,

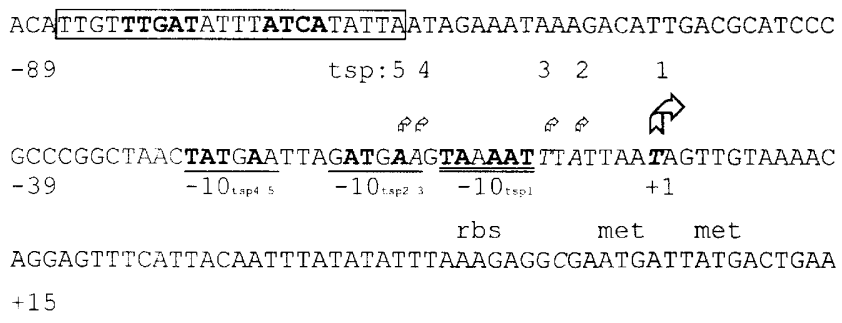

Fig. 1. Nucleotide sequence of the $h l y E$ promoter region. The following features are indicated: the FNR-binding site (boxed); three - 10 promoter elements (single and double underlined, consensus bases in bold); five transcript start points (arrowed, the major tsp, tsp1, is indicated by +1 and a large arrow); the ribosome-binding site (rbs) and two possible initiating methionine (met) residues.
JRG1728 and the same strain carrying plasmids expressing fnr or blyX (Table 1). In rich medium, expression of $h l y E$ was anaerobically induced 32 -fold in cultures expressing HlyX, but only ninefold by those expressing FNR, relative to aerobic expression in JRG3702. However, in the absence of either regulator (JRG3702), after growth on rich or minimal medium, a 5.7-fold anaerobic induction of $h l y E$ expression was still observed. Therefore, using the anaerobic expression of $h l y E$ in JRG3702 $(\Delta f n r)$ as a baseline, the FNRdependent anaerobic induction of $h l y E$ was only $1 \cdot 5$-fold compared to a HlyX-mediated induction of $5 \cdot 6$-fold. Growth in the presence of $30 \mathrm{mg} \mathrm{IPTG}^{-1}$ increases the cellular content of the regulators (FNR and HlyX) and resulted in a small increase in FNR-dependent blyE expression (265 Miller units without IPTG compared to 390 Miller units in the presence of IPTG), but the addition of IPTG increased HlyX-dependent expression to 2540 Miller units from 950 Miller units observed in the absence of IPTG, equivalent to an overall HlyXmediated 15-fold induction relative to JRG3702 (Table 1). Thus, it is clear that HlyX is a more efficient anaerobic activator of $h l y E$ transcription than FNR.

The lac $Z$ fusion data were consistent with the production of haemolysin by the three strains. After anaerobic growth on blood agar without added IPTG, only the hlyX-expressing strain, JRG2269, displayed the haemolytic phenotype, whereas in the presence of IPTG, low levels of haemolysin were produced by the $f n r-$ expressing strain, JRG3350. Irrespective of IPTG addition, JRG1728 ( $\Delta f n r)$ did not produce detectable levels of haemolysin.

Analyses of the FNR- $b l y E$ and HlyX-blyE DNase I footprints revealed similar protection patterns. Both regulators occupied the region between -97 and -61 (Fig. 2, lanes 2 and 3). A hypersensitive site at position -71 indicates that both FNR and HlyX distort the DNA in a similar manner. In the absence of FNR or HlyX, addition of $1 \mathrm{U}$ RNA polymerase caused the protection of a region between -83 and +5 (Fig. 2, lane 4). Hypersensitive bases at -41 and -42 were present, indicative of DNA distortion in the blyE-RNA polymerase binary complex. However, both FNR and HlyX were able to recruit RNA polymerase to the $h l_{y} E$

Table 1. Anaerobic activation of $h l y E$ expression by FNR and HlyX

FNR- and HlyX-driven $h l y E$ promoter activities were estimated by measuring $\beta$-galactosidase activity after growth of JRG3702 ( $\triangle$ fnr blyE::lacZ) and with transformants containing a second plasmid expressing either FNR (pGS330) or HlyX (pGS415) in the absence or presence of $30 \mathrm{mg}$ IPTG $1^{-1}$. The values quoted are the mean of duplicate assays from three independent cultures.

\begin{tabular}{|lccc|}
\hline \multirow{2}{*}{ Strain } & \multicolumn{3}{c|}{$\boldsymbol{\beta}$-Galactosidase activity (Miller units) } \\
\cline { 2 - 4 } & Aerobic & Anaerobic & Anaerobic + IPTG \\
\hline JRG3702 $(\Delta f n r)$ & 30 & 170 & 120 \\
JRG3653 $(f n r)$ & 37 & 265 & 390 \\
JRG3654 $(b l y X)$ & 50 & 950 & 2540 \\
\hline
\end{tabular}


$\begin{array}{llllllll}1 & 2 & 3 & 4 & 5 & 6 & 7\end{array}$

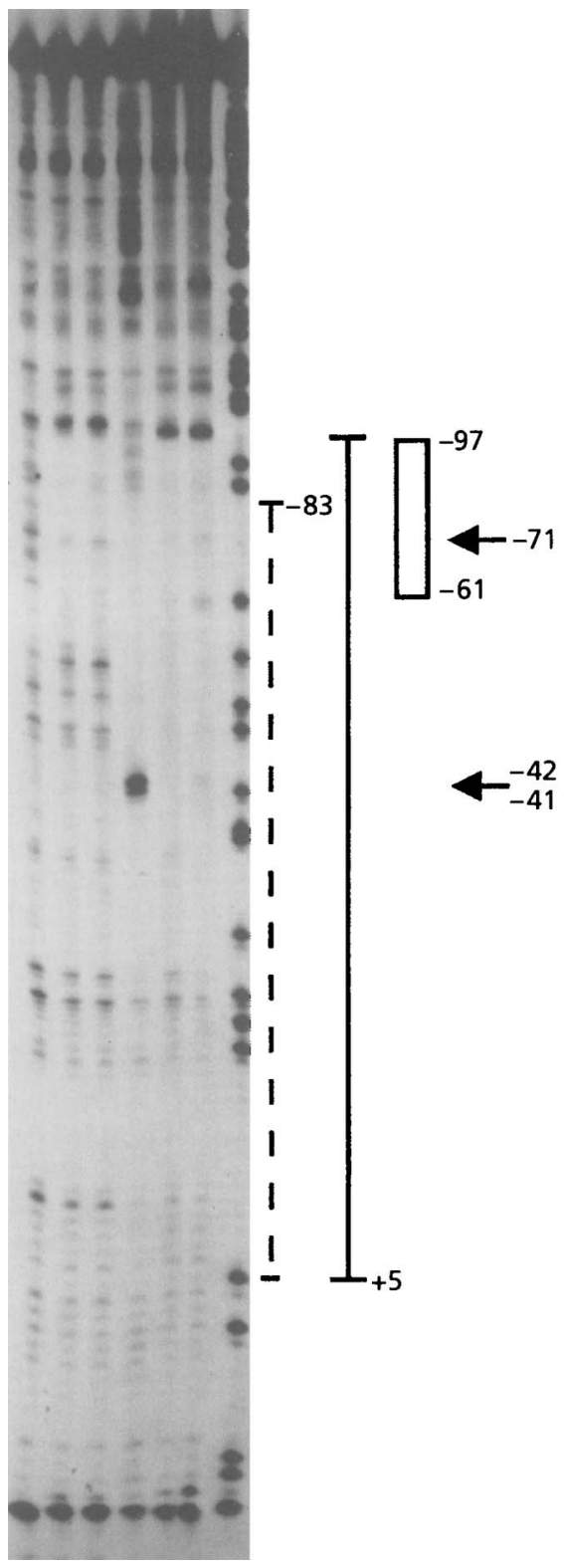

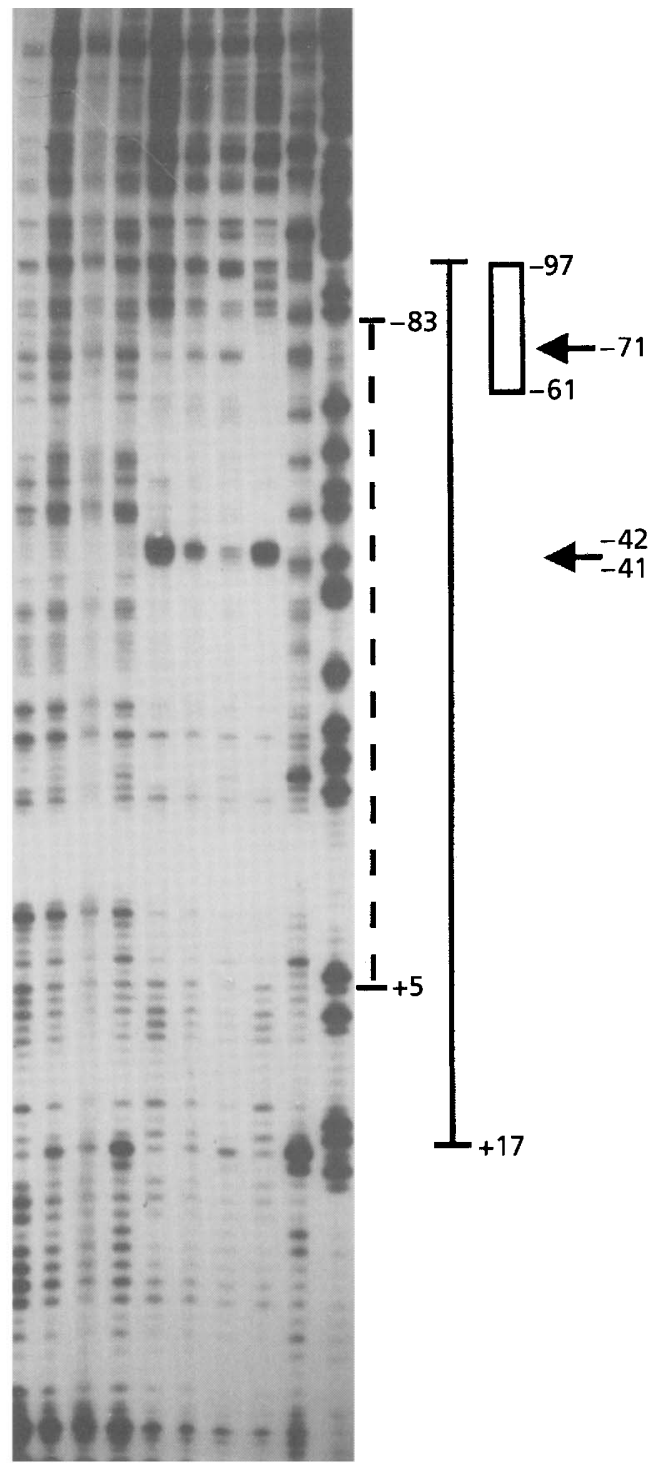

Fig. 2. DNase I footprints of the hlyE promoter region. Lanes: 1,8 and 10 , no protein: 2 , FNR $(100 \mathrm{nM}) ; 3, \mathrm{HlyX}(100 \mathrm{nM})$; 4, RNA polymerase $(1 \mathrm{U}, 100 \mathrm{nM})$; 5, RNA polymerase $(1 \mathrm{U})$ plus FNR $(100 \mathrm{nM})$; 6 , RNA polymerase $(1 \mathrm{U})$ plus HlyX $(100 \mathrm{nM}) ; 7$ and 17, calibrating Maxam \& Gilbert G tracks; 9, FNR (15 nM); 11, HlyX (15 nM); 12, FNR (15 nM) plus RNA polymerase $(0.1 \mathrm{U}, 10 \mathrm{nM}) ; 13$, FNR $(30 \mathrm{nM})$ plus RNA polymerase $(0.1 \mathrm{U}) ; 14$, HlyX $(15 \mathrm{nM})$ plus RNA polymerase $(0.1 \mathrm{U})$; 15 , RNA polymerase $(1 \mathrm{U}) ; 16$, RNA polymerase $(0 \cdot 1 \mathrm{U})$. The regions of FNR or HlyX (open box), RNA polymerase (dashed line), HlyX (FNR) plus RNA polymerase (solid line) protection and some hypersensitive positions (arrows) are indicated.

promoter because at concentrations insufficient to produce protection when present individually, FNR or Hly X $(15 \mathrm{nM})$ in combination with RNA polymerase $(0 \cdot 1 \mathrm{U})$, generated a region of protection from -97 to at least +5 corresponding to $h l y E-$ RNA polymerase-FNR (-HlyX) ternary complexes (Fig. 2, lanes 12-14). Although the protection of the FNR site was not as strong as that observed when higher concentrations of regulator were used (Fig. 2, lanes 5 and 6), these observations indicate that RNA polymerase and FNR (and HlyX) may bind co-operatively. The regions of protection for the FNR- and HlyX-containing ternary complexes were not identical. The HlyX-containing ternary complex protected the hlyE promoter from -97 to +17 compared to -97 to +5 for the equivalent FNR-containing complex. Increasing the concentration of FNR did allow the formation of a complex similar to that formed with HlyX (Fig. 2, lanes 5, 6 and 13). These observations indicate that HlyX is probably better able to direct RNA polymerase to the optimal position for transcription to 

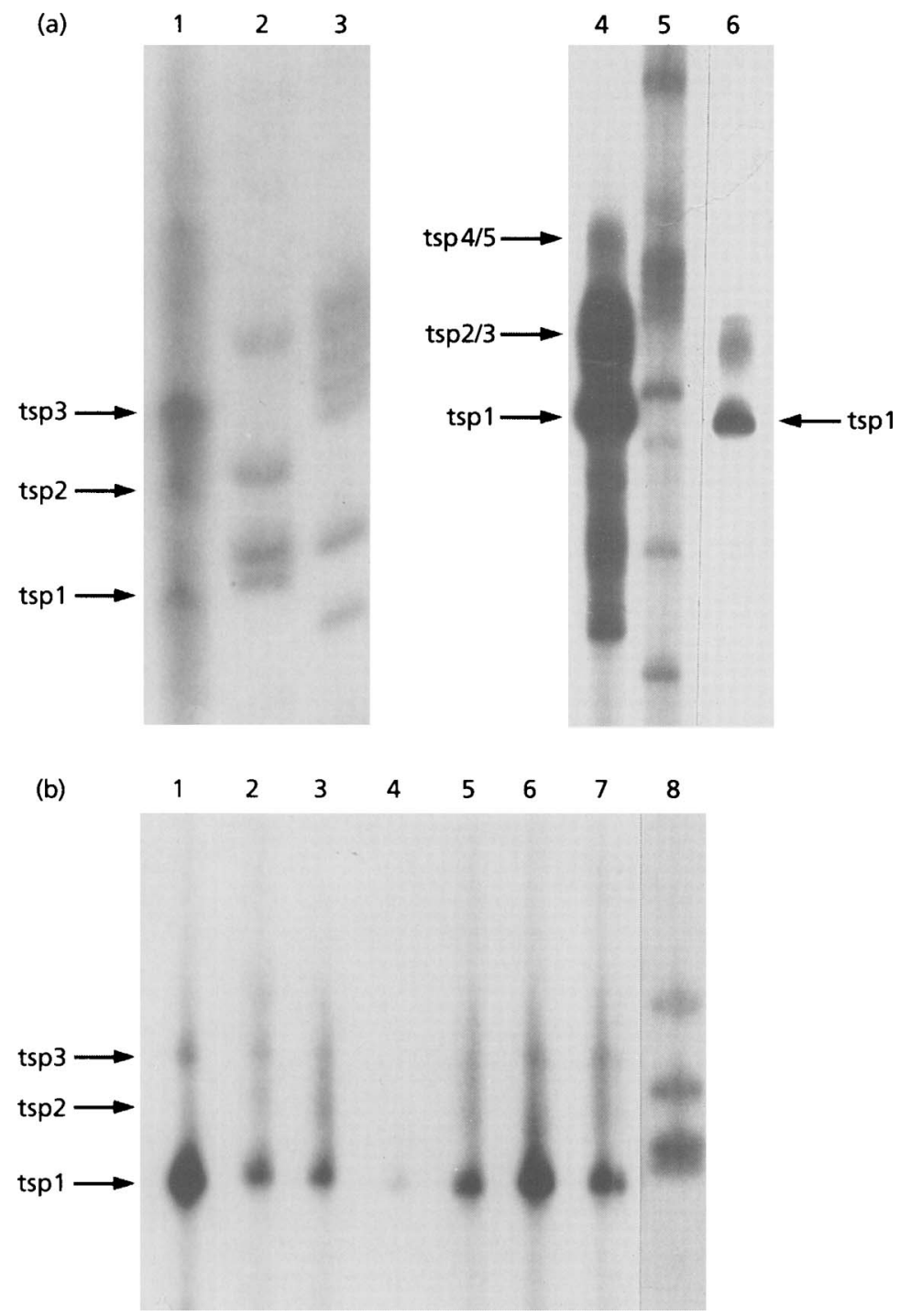

Fig. 3. (a) Location of transcript start sites for the hlyE promoter driven by FNR or HlyX. The 5 ' ends of the transcripts were determined by primer extension analysis and comparing the resulting molecules (lane 1 , FNR-expressing cultures; lanes 4 and 6 , HlyXexpressing cultures, lane 6 is a shorter exposure time of the sample in lane 4 to illustrate that tsp1 is the major transcript start) with calibrating Maxam \& Gilbert $G$ tracks (lane 2, yfiD DNA; lanes 3 and 5, ndh DNA). The transcript start points (tsp1-5) are indicated. (b) Location of hlyE transcript starts in a variety of genetic backgrounds. The $5^{\prime}$ ends of the transcripts were estimated by primer extension, all strains (except lanes 1 and 4) expressed fnr from the chromosome and the cultures were grown anaerobically for $24 \mathrm{~h}$. Lanes: 1, multicopy fnr; 2, wild-type; 3, rpos; 4, fnr; 5, ihfA; 6, fis; 7, arcA; 8, calibrating Maxam \& Gilbert $G$ track of yfiD DNA (Green \& Baldwin, 1997). occur. This re-alignment of polymerase is accompanied by the reduction/loss of hypersensitivity at positions -41 and -42 . However, transcription in vitro with $\sigma^{70}$ RNA polymerase indicated that, even in the presence of FNR or HlyX, the $h l y E$ transcript was not detectable (not shown), indicating that the ternary complexes formed are not transcriptionally competent.

\section{The hlyE promoter is a Class I FNR-dependent promoter}

Further analysis of the $h l y E$ promoter region revealed the presence of a ribosome-binding site (7 out of 9 consensus match, TAAAGAGGC) but several possible -10 elements (Fig. 1). Transcript mapping by primer extension with RNA isolated from the $h l y X$-expressing strain JRG2269 indicated that the blyE transcript is initiated from three positions: the major transcript start places the FNR site at -78.5 (tsp1); the minor transcripts place the FNR site at -73.5 (tsp2) and -71.5 (tsp3), respectively (Fig. 3a). The major transcript represented $80 \%$ of the total $h l y E$ message in this strain. The same three transcript start points were observed in an $f n r$-expressing strain although the relative proportions of the transcripts differed (Fig. 3a).

Overexposure of the autoradiographs allowed the detection of two additional minor $(\leqslant 1 \%$ of the total transcripts) transcript starts, tsp 4 and tsp5, which placed the FNR box at -63.5 and -62.5 , respectively. Therefore, as the position of the FNR-binding site at the blyE promoter is always beyond -41 , blyE is a Class I promoter (Ebright, 1993).

Expression of hlyE resembled that observed for a wildtype (fnr-expressing) strain in $r p o S$, ibfA, fis and $\operatorname{arcA}$ strains as judged by transcript mapping (Fig. 3b). However, in the fnr strain, JRG1728, the amount of blyE transcript (tsp1) produced was reduced by $12 \cdot 5$ fold, relative to the wild-type, but was still detectable. This apparent FNR-independent $h l y E$ transcription may account for the FNR-independent anaerobic induction observed in the blyE::lacZ reporter experiments (see 
Table 2. Anaerobic activation of the FF series and hlyE promoters by FNR and HlyX variants

The effect of various amino acid substitutions on anaerobic activation of FNR-dependent promoters. Promoter activity was estimated by measuring $\beta$-galactosidase activity after anaerobic growth of JRG1728 carrying the indicated pRW50 derivatives which encode the lac operon under the control of the following FNR-dependent promoters: FFpmelR, FNR site at -41.5 ;

$F F+20 p m e l R$, FNR site at $-61.5 ; F F-71.5 p m e l R$, FNR site at -71.5 and an improved -35

element; hlyE. FNR, HlyX and the derivatives were introduced on a second plasmid encoding FNR (pGS330), HlyX (pGS415), FNR-R179Q (pGS1053), FNR-F181Y (pGS1054), FNR-Q183A (pGS1055), FNR-R184P (pGS1056), FNR-P188A (pGS1057), FNR-A225T (pGS1086) or HlyX-A187P (pGS1003). The values quoted are the mean from duplicate assays of three independent cultures and the figures in parentheses represent the activity of the indicated FNR derivative relative to FNR.

\begin{tabular}{|c|c|c|c|c|}
\hline \multirow[t]{2}{*}{ FNR protein } & \multicolumn{4}{|c|}{$\beta$-Galactosidase activity (Miller units) } \\
\hline & FFpmelR & $F F+20 p m e l R$ & $F F-71.5 p m e l R$ & $h l y E$ \\
\hline FNR & $3800(1 \cdot 00)$ & $130(1.00)$ & $1600(1.00)$ & $275(1.00)$ \\
\hline FNR-R179Q & $3800(1.00)$ & $260(2 \cdot 00)$ & $1820(1 \cdot 13)$ & $285(1 \cdot 04)$ \\
\hline FNR-F181Y & $3850(1 \cdot 01)$ & $290(2 \cdot 23)$ & $2310(1 \cdot 44)$ & $360(1 \cdot 31)$ \\
\hline FNR-Q183A & $4135(1 \cdot 09)$ & $270(2 \cdot 07)$ & $2295(1.43)$ & $275(1.00)$ \\
\hline FNR-R184P & $1030(0 \cdot 27)$ & $150(1 \cdot 15)$ & $1070(0 \cdot 66)$ & $185(0.67)$ \\
\hline FNR-P188A & $4025(1.06)$ & $305(2 \cdot 34)$ & $2680(1.68)$ & $360(1 \cdot 31)$ \\
\hline FNR-A225T & $5380(1 \cdot 42)$ & $570(4 \cdot 36)$ & $3290(2 \cdot 05)$ & $585(2 \cdot 13)$ \\
\hline HlyX-A187P & $800(0 \cdot 21)$ & $200(1.53)$ & $1550(0 \cdot 96)$ & $510(1.85)$ \\
\hline HlyX & $1920(0 \cdot 51)$ & $770(5.92)$ & $3330(2 \cdot 08)$ & $950(3 \cdot 45)$ \\
\hline
\end{tabular}

above). Quantitative densitometry indicated that anaerobic cultures expressing HlyX produced at least 20 times more blyE transcripts than those expressing FNR in multicopy or from the chromosome.

\section{The region encompassing $\beta_{9}$ and $\beta_{10}$ of FNR contributes to FNR activation of Class I promoters}

The FNR and HlyX proteins bind to the same site at the Class I $h l y E$ promoter and initiate transcription from the same positions. However, only HlyX efficiently induces haemolysin production, indicating that the quality of the HlyX AR1 contacts with RNA polymerase are likely to be superior to those of FNR at the $b l y E$ and other Class I promoters (Green \& Baldwin, 1997). The AR1 of HlyX has been partially defined by the substitution A187P and lies in the loop between $\beta_{9}$ and $\beta_{10}$ (Green $\&$ Baldwin, 1997). This region of FNR was analysed using site-directed mutagenesis to substitute singly those amino acids unique to FNR with those present in HlyX (Fig. 4) and then testing the ability of the resulting proteins to activate transcription from the hlyE promoter and the model, semi-synthetic Class I $(F F+20 p m e l R$ and $F F-71.5 p m e l R)$ and Class II (FFpmelR) promoters.

Most of the substitutions made in the $\beta_{9}-\beta_{10}$ region had some effect on transcription activation from the Class I model promoters but little effect on the Class II FFpmelR promoter (Table 2). Such FNR variants displayed enhanced activation from Class I promoters compared with FNR. The FNR variant FNR-P188A was the most like HlyX in its regulation of Class I promoters but although it was some $2 \cdot 3$-fold better than FNR in activating transcription from $F F+20 p m e l R$ it was still poorer than HlyX (sixfold better than FNR). Studies with the blyE: lacZ reporter plasmid indicated that the replacement of FNR residues F181 or P188 with the amino acids found in the equivalent positions in HlyX increased blyE expression to 1.3 times that observed with FNR (Table 2), indicating that this region of FNR does contribute toward transcription activation at the blyE promoter. Interestingly, substitution of R184 of FNR by $\mathrm{P}$ (found in the equivalent position in HlyX) resulted in an FNR protein, FNR-R184P, which was defective in activation from the Class II promoter FFpmelR but was much less affected in the ability to activate transcription from Class I promoters. This contrasts with the transcriptional activities of the other FNR variants in the $\beta_{9}-\beta_{10}$ region which displayed increased activity at Class I promoters and unaltered activity at the Class II promoter, suggesting that the AR1 contacts at Class I and Class II promoters are not identical.

None of the substitutions in the $\beta_{9}-\beta_{10}$ region of FNR was sufficient to confer a haemolytic phenotype upon $E$. coli JRG1728.

The $\beta_{9}-\beta_{10}$ region of HlyX has been shown to be involved in transcription activation from Class I promoters and the substitution $\mathrm{A} 187 \mathrm{P}$ reduced activation from two model Class I promoters (Table 2, Green \& Baldwin, 1997). Accordingly, for bacteria expressing HlyX-A187P, anaerobic expression of $b l y E$ was reduced to only $54 \%$ of that observed with HlyX (Table 2). 


\section{The C-terminal element required for the expression of HlyE is defined by the substitution A255T}

The failure to generate an FNR protein with the capacity to confer a haemolytic phenotype on E. coli by making substitutions in the $\beta_{9}-\beta_{10}$ region was not surprising. Even though these variants increased blyE expression up to $1 \cdot 3$-fold relative to FNR, observation of the haemolytic phenotype is known to require elements of both the $\mathrm{N}$ - and C-terminal regions of HlyX (Green et al., 1992) and the $\beta_{9}-\beta_{10}$ region represents only the $\mathrm{N}$ terminal element. Thus the contribution of the $\beta_{11}-\beta_{12}$ region of FNR/HlyX was investigated. Position 225 (A in FNR, T in HlyX) was targeted because it is the only non-conservative substitution in $\beta_{11}$ (Fig. 4). Substitution of A225 of FNR with $T$ had dramatic effects on transcription activation from model promoters (Table 2). The altered FNR (FNR-A225T) combined the transcriptional activation properties of FNR at Class II promoters and HlyX at Class I promoters. Also, it allowed increased blyE expression and conferred a haemolytic phenotype upon JRG1728 $(f n r)$. Thus, it is likely that A225 is part of AR1 of FNR and represents the C-terminal element required for the anaerobic induction of haemolytic activity in E. coli $\mathrm{K}-12$ strains (Green et al., 1992), the $\mathrm{N}$-terminal element being previously defined as part of the $\beta_{9}-\beta_{10}$ loop (Green \& Baldwin, 1997).

The C-terminal regions of FNR and HlyX are rather different as FNR has an extra 9 aa. Investigation of the contribution of this region was attempted by generating two FNR proteins in which 17 and $10 \mathrm{C}$-terminal amino acids were absent. Unfortunately, both altered FNR proteins were insoluble and their transcription activation properties were therefore difficult to study. Neither derivative could anaerobically activate expression from $h l y E$ or any of the model promoters at $37^{\circ} \mathrm{C}$, even in the presence of IPTG. Consequently they did not confer a haemolytic phenotype at this temperature. However, if FNR-Cdel10-expressing cultures were stored at $4{ }^{\circ} \mathrm{C}$ for $14 \mathrm{~d}$ after anaerobic incubation for $24 \mathrm{~h}$ at $37^{\circ} \mathrm{C}$ on blood agar, the haemolytic phenotype was detected. An FNR-expressing control culture was haemolysin-negative.

\section{DISCUSSION}

Following analysis of the relative efficiencies of FNRand HlyX-mediated activation of model Class I and Class II promoters it was predicted that the $h l y E$ gene would possess an FNR site positioned at or beyond -61.5 , i.e. $h l y E$ would be expressed from an FNRdependent Class I promoter (Green \& Baldwin, 1997). The present work shows that this is the case with the major transcript initiating $78.5 \mathrm{bp}$ downstream of the FNR site. Thus, the molecular basis of the ability of HlyX but not FNR to confer a haemolytic phenotype upon $E$. coli probably lies in the relative efficiencies of FNR- and HlyX-mediated activation from Class I promoters. Studies with model promoters indicated that FNR is unable to activate transcription when positioned beyond -61.5 unless an improved -35 element is present in the promoter (Wing et al., 1995). The -35 element of $h l y E$ is very poor (GCTAAC, having only one base in common with the consensus) and thus it is not surprising that FNR is a weak activator of blyE expression. HlyX-mediated activation of Class I promoters is better than that of FNR because HlyX makes an enhanced AR1 contact with RNA polymerase (Green \& Baldwin, 1997). The AR 1 of HlyX was characterized by the mutation A187P which lies in the loop between $\beta_{9}$ and $\beta_{10}$. This region is equivalent to the AR1 of CRP and may be extended at least as far as the $\alpha_{\mathrm{D}}-\beta_{9}$ loop of HlyX (Green \& Baldwin, 1997). The role of this $\beta_{9}-\beta_{10}$ region of the CRP/FNR family of transcription regulators in activation from Class I promoters was confirmed by making individual replacements of the amino acids found in FNR with those of HlyX. As a result, in most cases FNR became more HlyX-like in its ability to activate expression of model Class I promoters and $h l y E$ itself. The exception was FNR-R184P which displayed much reduced activity at Class II promoters but was less compromised at Class I and blyE promoters. The AR1 contact between regulator RNA polymerase is a feature of both Class I and Class II promoters (Savery et al., 1996). For CRP at Class II promoters the role of the AR1 contact is that of anti-inhibition (Niu et al., 1996), whereas at Class I promoters AR1 makes an activating contact with the $\alpha$ subunit of RNA polymerase (Ebright, 1993). The results obtained with FNR-R184P suggest that the AR 1 contact at Class I promoters (FNR contacts the downstream $\alpha$ subunit of RNA polymerase) may not be identical to that at Class II promoters (FNR contacts the upstream $\alpha$ subunit) and that R184 may be more involved in making an anti-inhibition (Class II) rather than an activating contact (Class I). It has been suggested that the AR1 interaction between CRP and RNA polymerase is different at Class I and Class II promoters (Zhou et al., 1994). An inferior anti-inhibition contact provides a plausible explanation for the relatively poor performance of HlyX at Class II promoters (Green \& Baldwin, 1997).

None of the replacements in the $\beta_{9}-\beta_{10}$ region enabled FNR to induce a haemolytic phenotype in E. coli, nor did they increase $h l y E$ expression to the levels observed with HlyX. This indicated that there were other regions of the protein involved in activating transcription from Class I promoters. Replacement of A225 in $\beta_{11}$ of FNR with the equivalent T of HlyX indicated that AR1 may extend into $\beta_{11}$. This substitution allowed the altered FNR to confer the haemolytic phenotype. Even more striking were the effects on transcription activation from a set of model promoters. The A225T substitution generated an FNR which combined the transcription activation properties of FNR and HlyX. Thus, the AR1 of FNR/HlyX extends at least from $\beta_{9}-\beta_{10}$ through to $\beta_{11}$. These results indicate that FNR has a much larger AR1 surface for contacting the $\alpha$ subunit of RNA polymerase compared to CRP (Niu et al., 1994; Zhou et al., 1994). Another obvious region in which FNR and HlyX differ is at their C termini where FNR has a 9 aa 


\begin{tabular}{|c|c|c|}
\hline & $---\beta_{9}----\beta_{10}$ & $-\beta_{11-----\beta_{12}--}$ \\
\hline & $\underset{\downarrow \downarrow \downarrow \downarrow}{R P F A Q R G F S P}$ & 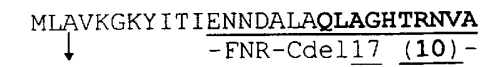 \\
\hline $\mathrm{YX}$ & QRYAAPGESA & MITVNGKYITINRMDELTV - \\
\hline & 188 & 234 \\
\hline
\end{tabular}

Fig. 4. Amino acid sequence of the $\beta_{9}-\beta_{10}$ and $\beta_{11}-\beta_{12}$ regions of FNR and HlyX. The amino acids selected for replacement are arrowed, the deleted C-terminal region of FNR is underlined and the numbering is for FNR.

extension. Two FNR proteins (FNR-Cdel17 and FNRCdel10) which lack 17 and 10 C-terminal amino acids, respectively, were insoluble at $37^{\circ} \mathrm{C}$ and were thus unsuitable for rigorous characterization, but it seems clear that the C-terminal region of FNR inhibits blyE expression at lower temperatures and is required for solubility and correct folding at $37^{\circ} \mathrm{C}$, even though it is absent from HlyX. It is possible that the C-terminal extension present in FNR acts to mask AR1, preventing efficient AR1: $\alpha$ contacts but, because of the low solubility of the C-terminally deleted FNRs, it has not been possible to investigate this further.

Reports in the literature indicate that $\operatorname{cly} A(h l y E)$ can be expressed in strains of $E$. coli which lack the histone-like protein HNS (Oscarsson et al., 1996). The transcript mapping presented here shows that the other histonelike proteins, integration host factor (IHF) and factor for inversion stimulation (Fis) do not affect $b l y E$ expression under anaerobic conditions. The sly $A$ gene product is also thought to regulate $c l y A(h l y E)$ expression but its mechanism of action and binding site are unknown (Oscarsson et al., 1996). It is possible that FNR (HlyX) and SlyA act in concert to maximize blyE expression and that HlyX-SlyA contacts may be important and more productive than the equivalent FNRSlyA contacts. There is, however, more to the endowment of a haemolytic phenotype than the production of the haemolysin polypeptide and other gene products may be required to allow efficient export of the haemolysin. SlyA, and indeed FNR and HlyX, may have a role in the regulation of these processes.

In conclusion, analysis of FNR- $h l y E$ and HlyX-blyE interactions indicates that $b l y E$ is a Class I FNRactivated promoter but, even though both regulators are able to recruit RNA polymerase, only HlyX positions RNA polymerase optimally to allow transcription. This suggests that the AR1 of HlyX, with a crucial contribution from T224, is superior to that of FNR. An obvious implication of these results is that a single substitution in FNR (or other transcription regulators) may be sufficient to permit the expression of otherwise silent virulence genes. It will be interesting to investigate AR1 of FNR proteins isolated from pathogenic strains of $E$. coli to establish if they possess AR1 surfaces that resemble that of FNR from E. coli K-12 or that of HlyX.

\section{ACKNOWLEDGEMENTS}

We would like to thank J.R. Guest for many useful discussions, S. J. W. Busby for his gift of pRW50, R. P. Gunsalus and R. Johnson for the ibfA and fis strains and A. J. Moir for DNA sequencing. This work was supported by the BBSRC and the Royal Society.

\section{REFERENCES}

Aiba, H., Adhya, S. \& Decrombrugghe, B. (1981). Evidence for two functional gal promoters in intact Escherichia coli cells. $J$ Biol Chem 256, 1905-1910.

Ebright, R. H. (1993). Transcription activation at Class I CAPdependent promoters. Mol Microbiol 8, 797-802.

Gerischer, V. \& Durre, P. (1992). Messenger-RNA analysis of the adc gene region of Clostridium acetobutylicum during the shift to solventogenesis. J Bacteriol 174, 426-433.

Green, J. \& Baldwin, M. L. (1997). HlyX, the FNR homologue of Actinobacillus pleuropneumoniae, is a $[4 \mathrm{Fe}-4 \mathrm{~S}]$ containing oxygen-responsive transcription regulator that anaerobically activates FNR-dependent Class I promoters via an enhanced AR1 contact. Mol Microbiol 24, 593-605.

Green, J., Trageser, M., Six, S., Unden, G. \& Guest, J. R. (1991). Characterization of the FNR protein of Escherichia coli, an iron binding transcriptional regulator. Proc R Soc Ser B 244, 137-144.

Green, J., Sharrocks, A. D., Maclnnes, J. I. \& Guest, J. R. (1992). Purification of HlyX, a potential regulator of haemolysin synthesis, and properties of HlyX:FNR hybrids. Proc R Soc Ser B 248, 79-84.

Green, J., Bennett, B., Jordan, P., Ralph, E. T., Thomson, A. J. \& Guest, J. R. (1996a). Reconstitution of the [4Fe-4S] cluster in FNR and demonstration of the aerobic-anaerobic transcription switch in vitro. Biochem $J$ 316, 887-892.

Green, J., Irvine, A. S., Meng, W. \& Guest, J. R. (1996b). FNR-DNA interactions at natural and semi-synthetic promoters. Mol Microbiol 19, 125-137.

Green, J., Anjum, M. F. \& Guest, J. R. (1996c). The $n d b$ binding protein (Nbp) regulates the $n d h$ gene of Escherichia coli in response to growth phase and is identical to Fis. Mol Microbiol 20, 1043-1055.

Guest, J. R., Green, J., Irvine, A. S. \& Spiro, S. (1996). The FNR modulon and FNR-regulated gene expression. In Regulation and Gene Expression in Escherichia coli, pp. 317-342. Edited by E. C. C. Lin \& A. S. Lynch. Austin, TX: R. G. Landes \& Co.

Lazazzera, B. A., Beinert, H., Khoroshilova, N., Kennedy, M. C. \& Kiley, P. J. (1996). DNA-binding and dimerization of the $\mathrm{Fe}-\mathrm{S}$ containing FNR protein of Escherichia coli are regulated by oxygen. $J$ Biol Chem 271, 2762-2768.

Maclnnes, J. I., Kim, J. E., Lian, C.-J. \& Soltes, G. A. (1990). Actinobacillus pleuropneumoniae blyX gene homology with the fnr gene of Escherichia coli. J Bacteriol 172, 4587-4592.

Maxam, A. M. \& Gilbert, W. (1980). Sequencing end-labelled DNA with base-specific chemical cleavages. Methods Enzymol 65, 499-560.

Miller, J. H. (1972). Experiments in Molecular Genetics. Cold Spring Harbor, NY: Cold Spring Harbor Laboratory.

Niu, W., Zhou, Y., Dong, Q., Ebright, Y. \& Ebright, R. H. (1994). Characterisation of the activating region of Escherichia coli 
catabolite gene activator protein (CAP). I. Saturation and alaninescanning mutagenesis. J Mol Biol 243, 595-602.

Niu, W., Kim, Y., Tau, G., Heyduk, T. \& Ebright, R. H. (1996). Transcription activation at Class II CAP-dependent promoters: two interactions between CAP and RNA polymerase. Cell 87, 1123-1134.

Oscarsson, J., Mizunoe, Y., Uhlin, B. E. \& Haydon, D. J. (1996). Induction of haemolytic activity in Escherichia coli by the slyA gene product. Mol Microbiol 20, 191-199.

Ramos, H. C., Boursier, L., Moszer, I., Kunst, F., Danchin, A. \& Glaser, P. (1995). Anaerobic transcription activation in Bacillus subtilis: identification of distinct FNR-dependent and -independent regulatory mechanisms. EMBO J 14, 5984-5994.

Savery, N., Rhodius, V. \& Busby, S. (1996). Protein-protein interactions during transcription activation: the case of the Escherichia coli cyclic AMP receptor protein. Philos Trans $R$ Soc Lond B Biol Sci 351, 543-550.

Spiro, S. \& Guest, J. R. (1987). Regulation and overexpression of the fnr gene of Escherichia coli. J Gen Microbiol 133, 3279-3288.

Wing, H. J., Williams, S. M. \& Busby, S. J. W. (1995). Spacing requirements for transcription activation by Escherichia coli FNR protein. J Bacteriol 177, 6704-6710.

Zhou, Y., Merkel, T. \& Ebright, R. H. (1994). Characterization of the activating region of Escherichia coli catabolite gene activator protein (CAP). II. Role at Class I and Class II CAP-dependent promoters. J Mol Biol 243, 603-610.

Received 11 July 1997; revised 22 August 1997; accepted 28 August 1997. 